\title{
Predictors of heterogeneity in the first-line treatment of patients with advanced/metastatic gastric cancer in the U.S.
}

\author{
Thomas Abrams ${ }^{1} \cdot$ Lisa M. Hess ${ }^{2} \cdot$ Yajun Emily Zhu ${ }^{2} \cdot$ William Schelman ${ }^{3} \cdot$ Astra M. Liepa $^{2} \cdot$ Charles Fuchs $^{4}$
}

Received: 3 November 2017 / Accepted: 24 January 2018 / Published online: 1 February 2018

(c) The International Gastric Cancer Association and The Japanese Gastric Cancer Association 2018

\begin{abstract}
Background Patients with metastatic gastric cancer have a poor prognosis (5-year survival of less than 10\%). This study was designed to describe the treatment patterns of patients with gastric cancer and to understand the factors associated with treatment choices to inform evidence-based care.

Methods A retrospective observational study was conducted using two real-world databases to describe treatment trends and to quantify variability in treatment patterns of patients diagnosed with advanced/metastatic gastric cancer between 1/1/2007 and 9/30/2014 in the U.S. Heterogeneity was measured by the Herfindahl-Hirschman Index (HHI). Predictors (baseline clinical, treatment, and demographic variables) of treatment regimen choice were evaluated using logistic regression.

Results A total of 5772 patients with advanced/metastatic gastric cancer were included in this study [5044 from claims data and 728 from electronic medical records (EMR)]. Of the 5044 from claims data, 2457 had evidence of metastatic disease at diagnosis. Only the fluorouracil + oxaliplatin regimen exceeded $10 \%$ utilization in the first-line setting [claims metastatic (12.1\%), claims advanced (8.2\%), and EMR metastatic (16.6\%) cohorts]. The HHI demonstrated extreme heterogeneity $(0.14$ for first-line therapy and 0.13 for second-line therapy). Patient age and geographic region of residence were significantly associated with treatment choice across all three cohorts in the first-line setting $(p<0.05)$.

Conclusion Treatment of patients with gastric cancer was highly variable. Despite the availability of treatment guidelines, there is a lack of consistent treatment patterns. There is a need to improve evidence-based care for patients with gastric cancer.
\end{abstract}

Keywords Population characteristics $\cdot$ Stomach neoplasms $\cdot$ Drug therapy $\cdot$ Antineoplastic agents

\section{Introduction}

Gastric cancer is an uncommon cancer in the U.S., with approximately 28,000 cases diagnosed in 2017 [1]. Globally, gastric cancer is much more common with over 930,000 cases diagnosed annually [2]. Primarily due to the infrequency of gastric cancer diagnoses in the U.S., there is no standard screening program in place as in other countries

Lisa M. Hess

Hess_lisa_m@lilly.com

1 Dana-Farber Cancer Institute, Harvard Medical School, Boston, MA, USA

2 Eli Lilly and Company, Global Patient Outcomes and Real World Evidence, Corporate Center, DC 5220, Indianapolis, IN 46254, USA

3 Eli Lilly and Company, US Medical Affairs, Indianapolis, IN, USA

4 Yale Cancer Center, New Haven, CT, USA that have a higher incidence rate. Of patients with gastric cancer, $28 \%$ are diagnosed with regionally-advanced and $35 \%$ with metastatic disease [3]. For patients with metastatic disease, the prognosis for long-term survival is poor, with only $5.2 \%$ of patients living 5 years or more [3].

Treatment guidelines are available to support the evidence-based treatment of patients diagnosed with gastric cancer to ensure that patients receive the best care and may achieve the best possible outcomes from this disease. For example, the National Comprehensive Cancer Network (NCCN) publishes gastric cancer treatment guidelines for the U.S. These guidelines are updated regularly, so oncologists can identify evidence-based treatment options to provide quality care and optimize patient outcomes. As of October 2017, in the first-line setting, only fluoropyrimidine plus cisplatin (with trastuzumab for HER2 overexpressing metastatic adenocarcinoma) is supported by Category 1 evidence (i.e., based on high-level scientific evidence and has consensus from panel members) for the treatment of 
locally advanced, recurrent, or metastatic gastric cancer [4]. Numerous additional regimens and combinations are listed with Category 2A evidence (lower levels of evidence, but consensus that the regimen is appropriate). In the secondline setting, Category 1 regimens include ramucirumab plus paclitaxel, single-agent paclitaxel, single-agent docetaxel, single-agent irinotecan, and single-agent ramucirumab [4]. Despite the relatively few number of treatment regimens with high level of scientific evidence, many options are appropriate, and optimal treatment strategies have yet to be identified. The combination of a disease with a poor prognosis and many reasonable alternatives has in part led to a disease that is associated with very high treatment heterogeneity. There is little evidence to guide usage of any one regimen over another [5-7].

This study was designed to examine the treatment patterns in gastric cancer to ascertain the factors associated with treatment decisions and to quantify treatment heterogeneity. The goal of the research was to provide a framework of evidence which will ultimately lead to a more evidence-based approach to the treatment of patients with gastric cancer.

\section{Methods}

\section{Study design and data sources}

A retrospective observational study was conducted using de-identified electronic medical records (EMR) and claims data. Two data sources were used for this study: Truven Marketscan claims and IMS Oncology EMR. Both sources contain non-overlapping patient-level variables. Truven Health MarketScan ${ }^{\circledR}$ Commercial and Medicare supplemental databases are fully integrated patient-level databases containing inpatient, outpatient, drug, and lab data from commercial and employer-sponsored Medicare supplemental plans. The databases reflect the real-world healthcare experience of employees, retirees, and dependents covered by the health benefit programs of large employers. The data are collected from approximately 350 different insurance companies and third party administrators. Rigorous validation methods are utilized to ensure that claims and enrollment data are complete, accurate, and reliable. The EMR data are derived from primarily medium and large community-based oncology practices. Each practice utilizes an electronic patient record system capturing detailed, patient-level clinical data that are then de-identified, assigned a synthetic ID, and integrated into the warehouse. The IMS data through December 2014 include 81 oncology practices treating more than 870,000 cancer patients from all 50 states. Patient-level data include, but are not limited to: diagnosis (non-oncology as well as oncology diagnoses), cancer staging, patient demographics, lab results and vital signs, injectable and oral medications, dosing, and drug regimens and treatment intervals.

\section{Eligibility criteria}

The study population included gastric cancer patients (including gastroesophageal junction cases coded as gastric cancer) in the two databases. Eligible patients were diagnosed with gastric or gastroesophageal junction cancer (ICD-9-CM: 151.x) between January 1, 2007 and September 30, 2014 (two or more claims were required for the claims cohort to avoid rule-out diagnostic codes), were at least 18 years of age, and had evidence of receiving chemotherapy, targeted therapy, and/or biologic therapy. Patients with a history of chemotherapy and/or prior cancer diagnoses (e.g., breast, colorectal prostate, ovarian, and lung) were excluded. Patients with evidence of an ICD-9 code suggesting gastrointestinal stromal tumor (GIST, ICD-9-CM of 238.1), or who received rituximab or imatinib at any time were also excluded.

Due to the nature of gastric cancer, eligible patients may also have esophageal (e.g., ICD-9-CM 150.x) cancer codes. These suggest gastroesophageal junction cancers, which are often coded with both gastric and esophageal cancer codes. The first occurrence of either the 150.x or 151.x code was considered the index diagnosis.

Metastatic gastric cancer (mGC) was identified by American Joint Committee on Cancer (AJCC) stage IV disease or tumor-node-metastasis (TNM) staging M1 codes (EMR only) or by ICD-9-CM codes indicating distant metastases (EMR and claims data). Advanced disease was defined as either the presence of metastatic codes (EMR and claims data) or the absence of any surgical resection/excision procedures prior to or during the first chemotherapy treatment (claims data only), to exclude adjuvant and neoadjuvant therapies from the assessment.

Chemotherapy, biologic, and targeted agents were defined on the basis of evidence of relevant Health Care Common Procedure Coding System (HCPCS), Common Procedural Terminology (CPT), and International Classification of Disease (ICD)-9-CM procedure codes, as well as on certain ICD-9-CM diagnostic codes and administrative revenue codes.

\section{Statistical methods}

Treatment regimens were defined as the set of anti-cancer agents received within a 28-day period of treatment initiation. The line of therapy was advanced if either the patient had a 90-day or greater gap in therapy before re-initiation of treatment, or if there was a change in chemotherapy agents with or without a 90-day gap (e.g., new regimen of anti-cancer agents). The line of therapy did not advance for 
treatment switching between similar agents (e.g., fluorouracil and capecitabine) or the addition of a biologic/targeted agent to a chemotherapy regimen.

Treatment heterogeneity was evaluated using the Herfindahl-Hirschman Index $(H H I)$ using the following formula $[8,9]$ :

$H=\sum_{i=1}^{N} s_{i}^{2}$

where $s_{i}$ is the proportion of regimen $i$ in the line of therapy, and $N$ is the number of regimens. A lower HHI score indicates greater heterogeneity. Scores range from 0 to 1 ; close to zero indicates extreme heterogeneity, with a very wide variety of different treatment regimens being used, and 1.0 reflects complete homogeneity, with only a single regimen being used in an entire population. The HHI is a measure that has been used to evaluate health care market share [10, 11]. Treatment volume and heterogeneity were assessed using the HHI using the number of eligible patients identified by year in EMR by practice site; variables to identify the oncology site of practice are not present in claims data.

Predictors of treatment choice for the ten most common regimens observed in the data were evaluated using logistic regression with a stepwise variable selection procedure. Factors evaluated from claims data included HER2 testing, prior therapy received, comorbidities [including $H$ pylori infection and gastroesophageal reflux disease (GERD)], prescription burden, proton pump inhibitor use, gender, age, metastatic disease, primary tumor location, and geographic region. Factors studied from EMR included baseline clinical and demographic variables of gender, age, comorbidities from the Charlson comorbidity index (CCI), Eastern Cooperative Oncology Group (ECOG) performance status, disease stage at diagnosis, and geographic region. No imputation was made for missing variables in the model.

\section{Results}

\section{Patient cohort}

As demonstrated in Table 1, a total of 3185 patients with mGC met eligibility criteria: 2457 from the claims database and 728 from EMR data. When using the criteria for advanced disease, there were 5044 patients identified in the claims data. Patients were relatively similar across the EMR and claims databases and by advanced/metastatic definitions in claims data. All cohorts had a mean age of slightly over 60 years of age, the mean duration of follow-up in the data ranged from 316 to 374 days, and mean time from diagnosis to the start of first-line therapy ranged from 73 to 79 days. However, the EMR database was predominantly from the South region of the U.S. (61\%), whereas the claims database included $30-33 \%$ of the cohort from the South region.

\section{Treatment patterns and heterogeneity}

There were 228 unique treatment regimens identified in the first-line setting for $\mathrm{mGC}$ and 289 for the treatment of advanced disease in claims data. Concomitant radiation therapy was received by $296(12.0 \%)$ and 1103 (21.9\%) of patients in the metastatic and advanced claims cohorts, respectively. In the EMR data, 87 unique treatment regimens were used in the first-line setting for mGC. Many of these regimens were used by fewer than 30 patients $(<4 \%)$ in the cohort. As demonstrated in Table 2, 37 and 39\% of first-line regimens used in the metastatic claims and EMR cohorts, respectively, were categorized as preferred regimens in the NCCN guidelines, demonstrating that the vast majority of patients received non-preferred therapies. However, most patients with metastatic disease received first-line regimens with a fluoropyrimidine as either monotherapy or in combination with other agents (66.4\% in claims and $91.2 \%$ in EMR). It is important to note that oral medications (e.g., capecitabine) tend to be underreported in EMR data, and lack of a specific drug code (e.g., unclassified agents) is not uncommon for claims submitted for patients with gastric cancer.

In addition to the descriptive treatment patterns, treatment variability was high as demonstrated by the low HHI scores in claims data for the advanced/metastatic cohort over time (Table 3). Both first- and second-line therapy demonstrated very high heterogeneity (HHI scores had little variation, ranging from 0.13 to 0.20 for first-line therapy and from 0.11 to 0.17 in the second-line setting. In EMR data, heterogeneity at practice sites was high regardless of patient volume (Table 4). The site with the largest volume of patients had an HHI score of 0.10 in the first line and 0.05 is the second line, and the site with the smallest patient volume had an HHI score of 0.22 in the first line and 0.24 in the second line (for sites with at least 20 patients).

\section{Factors associated with treatment choice}

A total of 3105 patients in the advanced claims cohort had sufficient data to be included in the modeling analyses. Statistically significant factors associated with treatment choice in the first-line setting are summarized in Table 5. Factors that influenced treatment choice in both the advanced and metastatic disease settings in the claims cohort included patient age, concomitant medications, geographic region, HER2 testing, and diabetes (all $p<0.05$ ). There were 539 patients included in the modeling analyses from the EMR cohort; only age $(p=0.0003)$ and geographic 
Table 1 Patient characteristics

\begin{tabular}{|c|c|c|c|}
\hline Patients' characteristics & $\begin{array}{l}\text { Claims mGC cohort } \\
(N=2457)\end{array}$ & $\begin{array}{l}\text { Claims advanced GC } \\
\text { cohort }(N=5044)\end{array}$ & $\begin{array}{l}\text { EMR mGC } \\
\text { cohort } \\
(N=728)\end{array}$ \\
\hline Mean age (SD) & $60.4(11.9)$ & $62.3(12.2)$ & $62.7(12.1)$ \\
\hline Male, $n(\%)$ & $1758(71.6)$ & $3744(74.2)$ & $471(64.7)$ \\
\hline \multicolumn{4}{|l|}{ Geographic region, $n(\%)$} \\
\hline South & $799(32.5)$ & $1532(30.4)$ & $443(60.9)$ \\
\hline North central/midwest & $684(27.8)$ & $1360(27.0)$ & $68(9.3)$ \\
\hline Northeast & $416(16.9)$ & $868(17.2)$ & $124(17.0)$ \\
\hline West & $377(15.3)$ & $827(16.4)$ & $91(12.5)$ \\
\hline Unknown & $181(7.4)$ & $457(9.1)$ & $2(0.3)$ \\
\hline \multicolumn{4}{|l|}{ Plan type, $n(\%)$} \\
\hline Preferred provider organization & $1322(53.8)$ & $2569(50.9)$ & - \\
\hline Comprehensive & $480(19.5)$ & $1188(23.6)$ & - \\
\hline Health maintenance organization & $259(10.5)$ & $514(10.2)$ & - \\
\hline Non-capitated point of service (POS) plan & $161(6.6)$ & $309(6.1)$ & - \\
\hline Consumer-driven health plan & $61(2.5)$ & $117(2.3)$ & - \\
\hline Exclusive provider organization & $39(1.6)$ & $71(1.4)$ & - \\
\hline POS plan with capitation & $17(0.7)$ & $39(0.8)$ & - \\
\hline Missing/unknown & $118(4.8)$ & $237(4.7)$ & $728(100)$ \\
\hline $\begin{array}{l}\text { Total prescription burden at start of first-line therapy, mean (SD) number } \\
\text { of drugs }\end{array}$ & $4.5(4.8)$ & $4.6(5.0)$ & - \\
\hline Total duration of follow-up from index diagnosis, mean (SD) days & $316.2(281.0)$ & $370.9(335.1)$ & $374.2(337.0)$ \\
\hline Time from diagnosis to start of first-line therapy, mean (SD) days & $78.7(160.7)$ & $78.5(179.3)$ & $72.6(179.0)$ \\
\hline Charlson comorbidity index score, mean (SD) & $1.7(2.6)$ & $1.3(2.1)$ & - \\
\hline HER2 tested at first-line therapy, $n(\%)$ & $1323(53.8)$ & $2300(45.6)$ & - \\
\hline Proton pump inhibitor use, $n(\%)$ & $769(31.3)$ & $1538(30.5)$ & - \\
\hline \multicolumn{4}{|l|}{ ECOG performance status, $n(\%)$} \\
\hline 0 & - & - & $110(15.1)$ \\
\hline 1 & - & - & $144(19.8)$ \\
\hline 2 & - & - & $46(6.3)$ \\
\hline $3+$ & - & - & $7(1.0)$ \\
\hline Missing/unknown & $2457(100)$ & $5044(100)$ & $421(57.8)$ \\
\hline
\end{tabular}

$m G C$ metastatic gastric cancer, $E M R$ electronic medical records, $G C$ gastric cancer, $S D$ standard deviation, $P O S$ point of service, $E C O G$ eastern cooperative oncology group

region ( $p=0.0008)$ were factors associated with treatment choice for metastatic disease; however, several of the factors included in the model, such as ECOG performance status, had high levels of missing data in EMR (Table 1).

\section{Discussion}

Consistent with the previous research [7], treatment variability was high for all practice sites, geographic regions and by year of diagnosis. The prior descriptive work is enhanced by the use of the HHI score, an objective measurement of heterogeneity. In this study, HHI scores demonstrated very high variability. This heterogeneity may in part be due to the lack of defined optimal treatment strategies, particularly in the first-line setting. There remains a need to identify best treatment practices to ensure that care for patients is in accordance with treatment guidelines.

The factors consistently associated with treatment choice across all three analyses (claims advanced cohort, claims metastatic cohort, and EMR metastatic cohort) were patient age and geographic region. Geographic factors, rather than clinical factors, were also significantly associated with treatment choice in an EMR study evaluating the role of patientreported symptoms in treatment decisions [6]. However, the incorporation of claims data for this study enabled the identification of significant factors that are not present in EMR data, such as comorbid conditions, HER2 testing, and prescription burden. This suggests that while geographic variation still holds a key role in treatment decision making, 
Table 2 Treatment regimens used by $>30$ patients in at least one cohort

\begin{tabular}{|c|c|c|c|}
\hline Regimen & $\begin{array}{l}\text { Claims mGC cohort }(N=2457) \\
N(\%)\end{array}$ & $\begin{array}{l}\text { Claims advanced GC cohort } \\
(N=5044) \\
N(\%)\end{array}$ & $\begin{array}{l}\text { EMR mGC cohort } \\
(N=728) \\
N(\%)\end{array}$ \\
\hline \multicolumn{4}{|l|}{ First-line therapy } \\
\hline Capecitabine & $182(7.4)$ & $294(5.8)$ & $0(0.0)$ \\
\hline Capecitabine cisplatin epirubicin ${ }^{\mathrm{a}}$ & $21(0.9)$ & $35(0.7)$ & $1(0.1)$ \\
\hline Capecitabine epirubicin oxaliplatin ${ }^{\mathrm{a}}$ & $121(4.9)$ & $197(3.9)$ & $12(1.6)$ \\
\hline Capecitabine oxaliplatin $^{\mathrm{a}}$ & $34(1.4)$ & $52(1.0)$ & $6(0.8)$ \\
\hline Carboplatin docetaxel & $43(1.8)$ & $72(1.4)$ & $27(3.7)$ \\
\hline Carboplatin docetaxel fluorouracil $^{\mathrm{a}}$ & $15(0.6)$ & $34(0.7)$ & $5(0.7)$ \\
\hline Carboplatin fluorouracil paclitaxel & $9(0.4)$ & $34(0.7)$ & $7(1.0)$ \\
\hline Carboplatin paclitaxel & $144(5.9)$ & $548(10.9)$ & $69(9.5)$ \\
\hline Carboplatin paclitaxel unclassified & $55(2.2)$ & $175(3.5)$ & $0(0.0)$ \\
\hline Cisplatin & $19(0.8)$ & $47(0.9)$ & $4(0.5)$ \\
\hline Cisplatin docetaxel & $32(1.3)$ & $60(1.2)$ & $10(1.4)$ \\
\hline Cisplatin docetaxel fluorouracil $^{\mathrm{a}}$ & $160(6.5)$ & $222(4.4)$ & $56(7.7)$ \\
\hline Cisplatin docetaxel fluorouracil unclassified & $38(1.5)$ & $44(0.9)$ & $0(0.0)$ \\
\hline Cisplatin epirubicin fluorouracil ${ }^{\mathrm{a}}$ & $65(2.6)$ & $155(3.1)$ & $33(4.5)$ \\
\hline Cisplatin fluorouracil $^{\mathrm{a}}$ & $78(3.2)$ & $331(6.6)$ & $6(0.8)$ \\
\hline Cisplatin fluorouracil unclassified & $11(0.4)$ & $41(0.8)$ & $0(0.0)$ \\
\hline Cisplatin irinotecan & $46(1.9)$ & $81(1.6)$ & $20(2.7)$ \\
\hline Cisplatin paclitaxel & $16(0.7)$ & $31(0.6)$ & $4(0.5)$ \\
\hline Docetaxel & $34(1.4)$ & $54(1.1)$ & $8(1.1)$ \\
\hline Docetaxel fluorouracil oxaliplatin ${ }^{\mathrm{a}}$ & $57(2.3)$ & $93(1.8)$ & $20(2.7)$ \\
\hline Epirubicin fluorouracil oxaliplatin ${ }^{a}$ & $25(1.0)$ & $42(0.8)$ & $11(1.5)$ \\
\hline Epirubicin oxaliplatin & $66(2.7)$ & $125(2.5)$ & $63(8.7)$ \\
\hline Fluorouracil & $180(7.3)$ & $282(5.6)$ & $52(7.1)$ \\
\hline Fluorouracil oxaliplatin ${ }^{\mathrm{a}}$ & $297(12.1)$ & $415(8.2)$ & $121(16.6)$ \\
\hline Fluorouracil oxaliplatin trastuzumab ${ }^{\mathrm{a}}$ & $33(1.3)$ & $41(0.8)$ & $9(1.2)$ \\
\hline Fluorouracil oxaliplatin unclassified & $19(0.8)$ & $39(0.8)$ & $0(0.0)$ \\
\hline Oxaliplatin & $40(1.6)$ & $66(1.3)$ & $27(3.7)$ \\
\hline \multirow[t]{2}{*}{ Unclassified } & $83(3.4)$ & $469(9.3)$ & $0(0.0)$ \\
\hline & $\begin{array}{l}\text { Claims mGC cohort }(N=1317) \\
N(\%)\end{array}$ & $\begin{array}{l}\text { Claims advanced GC cohort } \\
(N=1714) \\
N(\%)\end{array}$ & $\begin{array}{l}\text { EMR mGC cohort } \\
(N=304) \\
N(\%)\end{array}$ \\
\hline \multicolumn{4}{|l|}{ Second-line therapy } \\
\hline Capecitabine & $69(5.2)$ & $95(5.5)$ & $2(0.7)$ \\
\hline Capecitabine epirubicin oxaliplatin & $38(2.9)$ & $58(3.4)$ & $5(1.6)$ \\
\hline Capecitabine oxaiplatin & $38(2.9)$ & $39(2.3)$ & $2(0.7)$ \\
\hline Carboplatin docetaxel & $18(1.4)$ & $31(1.8)$ & $9(3.0)$ \\
\hline Carboplatin paclitaxel & $57(4.3)$ & $94(5.5)$ & $21(6.9)$ \\
\hline Carboplatin paclitaxel unclassified & $18(1.4)$ & $31(1.8)$ & $0(0.0)$ \\
\hline Cisplatin docetaxel fluorouracil & $37(2.8)$ & $45(2.6)$ & $10(3.3)$ \\
\hline Cisplatin epirubicin fluorouracil & $18(1.4)$ & $30(1.8)$ & $11(3.6)$ \\
\hline Cisplatin irinotecan & $48(3.6)$ & $58(3.4)$ & $12(3.9)$ \\
\hline Docetaxel $^{\mathrm{a}}$ & $61(4.6)$ & $62(3.6)$ & $17(5.6)$ \\
\hline Fluorouracil & $56(4.3)$ & $78(4.6)$ & $11(3.6)$ \\
\hline Fluorouracil irinotecan & $62(4.7)$ & $69(4.0)$ & $21(6.9)$ \\
\hline Fluorouracil oxaliplatin & $116(8.8)$ & $138(8.1)$ & $22(7.2)$ \\
\hline Irinotecan $^{\mathrm{a}}$ & $55(4.2)$ & $59(3.4)$ & $14(4.6)$ \\
\hline Paclitaxel $^{\mathrm{a}}$ & $36(2.7)$ & $37(2.2)$ & $1(4.2)$ \\
\hline Unclassified & $31(2.4)$ & $100(5.8)$ & $0(0.0)$ \\
\hline
\end{tabular}

$m G C$ metastatic gastric cancer, $E M R$ electronic medical records, $G C$ gastric cancer

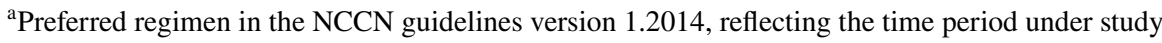


Table 3 Treatment heterogeneity over time

\begin{tabular}{lclll}
\hline $\begin{array}{l}\text { Year of } \\
\text { initiation of } \\
\text { therapy }\end{array}$ & First line $N^{\mathrm{a}}$ & $\begin{array}{l}\text { First- } \\
\text { line HHI } \\
\text { score }^{\mathrm{a}}\end{array}$ & $\begin{array}{l}\text { Second line } \\
N\end{array}$ & $\begin{array}{l}\text { Second-line } \\
\text { HHI score }\end{array}$ \\
\hline All years & 3105 & 0.14 & 1207 & 0.13 \\
2007 & 162 & 0.16 & 35 & 0.17 \\
2008 & 242 & 0.15 & 95 & 0.14 \\
2009 & 307 & 0.14 & 115 & 0.16 \\
2010 & 404 & 0.13 & 132 & 0.11 \\
2011 & 516 & 0.14 & 166 & 0.13 \\
2012 & 554 & 0.15 & 219 & 0.14 \\
2013 & 513 & 0.20 & 215 & 0.14 \\
2014 & 407 & 0.19 & 230 & 0.15 \\
\hline
\end{tabular}

HHI Herfindahl-Hirschman index

${ }^{a}$ Limited to patients in the advanced cohort. As a result, this excluded adjuvant/neoadjuvant therapies

Table 4 Treatment heterogeneity by site volume

\begin{tabular}{|c|c|c|c|c|}
\hline Site ID & $\begin{array}{l}\text { Number of } \\
\text { 1L metastatic } \\
\text { patients }\end{array}$ & 1L site $\mathrm{HHI}$ & $\begin{array}{l}\text { Number of } \\
2 \mathrm{~L} \text { patients }\end{array}$ & 2L site $\mathrm{HHI}$ \\
\hline 166 & 198 & 0.1035 & 205 & 0.0454 \\
\hline 58 & 94 & 0.0887 & 102 & 0.0656 \\
\hline 61 & 37 & 0.1804 & 61 & 0.1008 \\
\hline 84 & 23 & 0.1682 & 20 & 0.1150 \\
\hline 52 & 21 & 0.1020 & 20 & 0.1050 \\
\hline 164 & 21 & 0.1565 & 13 & 0.1243 \\
\hline 47 & 20 & 0.1200 & 29 & 0.0963 \\
\hline 267 & 18 & 0.2284 & 18 & 0.1049 \\
\hline 66 & 15 & 0.2533 & 16 & 0.1328 \\
\hline 25 & 14 & 0.1837 & 13 & 0.1124 \\
\hline 11 & 12 & 0.1806 & 12 & 0.1528 \\
\hline 281 & 12 & 0.1528 & 14 & 0.1327 \\
\hline 54 & 11 & 0.1736 & 11 & 0.1240 \\
\hline 2 & 10 & 0.2600 & 5 & 0.2800 \\
\hline 62 & 10 & 0.1800 & 16 & 0.1016 \\
\hline 85 & 6 & 0.3889 & 23 & 0.0813 \\
\hline 38 & 8 & 0.1875 & 15 & 0.1911 \\
\hline 7 & 9 & 0.1852 & 13 & 0.1124 \\
\hline 279 & 8 & 0.1875 & 12 & 0.1111 \\
\hline 13 & 3 & 0.3333 & 12 & 0.3056 \\
\hline 36 & 9 & 0.2840 & 11 & 0.1570 \\
\hline 41 & 6 & 0.2222 & 10 & 0.1800 \\
\hline 87 & 6 & 0.2222 & 10 & 0.2400 \\
\hline
\end{tabular}

Limited to sites with 10 or more patients in at least one line of therapy

$H H I$ Herfindahl-Hirschman index, $1 L$ first line, $2 L$ second line

important clinical factors such as HER2 status and patient health are incorporated into the decision between treatment alternatives.
While the value of real-world data includes a representation of actual treatment choices in an uncontrolled setting, the data sources to obtain these data are limited by the fact that they are collected for purposes other than research. EMR do not generally contain oral medications, which are recorded within pharmacy databases. As a result, the use of drugs such as capecitabine are underreported in EMR systems unless the provider manually enters this information. Claims have the advantage of collecting data regardless of the point of service, but are limited to those resources that are reimbursed by insurance. As demonstrated in this study, unclassified drug codes are entered frequently, making it impossible to know what medication is actually being used. This may underestimate heterogeneity, due to the consolidation of any number of different drugs in the 'unclassified' category. This research was conducted on databases that ended in 2015, which was shortly after the FDA approval of ramucirumab. Newer data on treatment patterns show that ramucirumab is frequently prescribed in the secondline setting; [5, 12]; however, the time frame of available data for this study does not account for this change in treatment patterns in the second-line setting. Other limitations of real-world data sources include the limited clinical details of the patient's disease (e.g., stage) in claims; therefore, assumptions were made to classify patients as 'metastatic' or 'advanced,' although it is known that the use of metastatic codes is incomplete in administrative claims data which can result in underestimation of these groups [13]. It is also possible misclassification could occur; however, the differences in chemoradiation therapy in the advanced (22\%) versus the metastatic cohorts (12\%), suggest that the rules applied to define these groups were directionally accurate. The high use of carboplatin + paclitaxel in all groups may be associated with radiation therapy. Future research may wish to consider excluding patients who receive chemoradiation from treatment patterns work; however, only claims are able to identify these patients, as radiation use is not recorded in IMS EMR. This remains a limitation for research using EMR that are not linked to other databases. Despite these limitations, large administrative and clinical databases provide an opportunity to retrospectively observe the care and outcomes of patients without the potential bias that may be incurred when it is known that the treatment and outcomes are being observed as in a prospective controlled research study.

While patients and providers benefit from having treatment choices, these decisions are better limited to those supported by scientific evidence as to their safety and clinical benefit. Given that the 2017 NCCN guidelines have been reduced since 2014 to include only six regimens with Level 1 evidence across the first two lines of therapy for advanced disease (fluoropyrimidine + cisplatin; ramucirumab + paclitaxel; single-agent docetaxel; single-agent paclitaxel; single-agent irinotecan; or single-agent ramucirumab) [4], it is likely that 
Table 5 Factors associated with treatment choice for advanced and metastatic disease in the first-line setting

\begin{tabular}{lc}
\hline Factors-claims advanced disease $(n=3105)$ & $p$ value \\
\hline Age at diagnosis & $<0.0001$ \\
Number of concomitant prescriptions & $<0.0001$ \\
Geographic region & $<0.0001$ \\
Location of primary cancer & $<0.0001$ \\
Proton pump inhibitor use, yes/no & 0.029 \\
HER2 testing, yes/no & $<0.0001$ \\
Evidence of metastatic disease, yes/no & $<0.0001$ \\
Chronic obstructive pulmonary disorder, yes/no & 0.038 \\
Diabetes, yes/no & 0.046 \\
Ulcer disease, yes/no & 0.0002 \\
\hline Factors-claims metastatic disease $(n=1602)$ & $p$ value \\
\hline Age at diagnosis & $<0.0001$ \\
Number of concomitant prescriptions & $<0.0001$ \\
Geographic region & $<0.0001$ \\
HER2 testing, yes/no & $<0.0001$ \\
Diabetes, yes/no & 0.0045 \\
\hline Factors-electronic medical records metastatic disease & $p$ value \\
$(n=539)$ & \\
\hline Age at diagnosis & 0.0003 \\
Geographic region & 0.0008 \\
\hline
\end{tabular}

of the majority of treatment currently given has lower levels of evidence or no scientific data to support its use, as was demonstrated in this study. Patient outcomes will be maximized when treatment choice allows for patient and provider flexibility, but is based on evidence-based care [14]. This study suggests that while some important clinical factors aid in the decision, there remains a need to further refine and streamline the care received by these patients. Evidence-based cancer care has long been a known need for improving cancer care in the U.S., and remains a clear need, particularly for gastric cancer patients.

Funding This study was an unfunded research project conducted with the material support in the form of employee time and data resources by Eli Lilly and Company.

\section{Compliance with ethical standards}

Conflict of interest LMH, YEZ, WS, and AML are employees of Eli Lilly and Company.

Ethical standards This study was deemed exempt from Institutional Review Board review in accordance with the US Code of Federal Regulations [45CFR46.101(b)] as these data do not contain any variables that could identify an individual subject either directly or indirectly.

\section{References}

1. Siegel RL, Miller KD, Jemal A. Cancer statistics, 2017. CA Cancer J Clin. 2017;67(1):7-30.

2. Torre LA, Bray F, Siegel RL, Ferlay J, Lortet-Tieulent J, Jemal A. Global cancer statistics, 2012. CA Cancer J Clin. 2015;65(2):87-108.

3. Cancer stat facts: stomach cancer, SEER 18 2007-2013. https ://seer.cancer.gov/statfacts/html/stomach.html.

4. NCCN Clinical practice guidelines in oncology, Gastric cancer version 4.2017 https://www.nccn.org/professionals/physi cian_gls/pdf/gastric.pdf.

5. Barzi A, Hess LM, Zhu YE, Liepa AM, Fang Y, Kuder C, Beyrer J, Chao J. Treatment and outcomes of patients with advanced gastric, esophageal, or gastroesophageal junction adenocarcinoma in the United States. Ann Oncol 2017;28(Suppl 3):35.

6. Hess LM, Cui ZL, Wu Y, Li X, Liepa AM, Abraham SM, Schelman W. Patient experience after receiving a diagnosis of gastric cancer in the USA. J Gastrointest Cancer. 2016. https://doi. org/10.1007/s12029-016-9904-7.

7. Hess LM, Michael D, Mytelka DS, Beyrer J, Liepa AM, Nicol S. Chemotherapy treatment patterns, costs, and outcomes of patients with gastric cancer in the United States: a retrospective analysis of electronic medical record (EMR) and administrative claims data. Gastric Cancer. 2016;19(2):607-15.

8. Weinstock DS. Using the Herfindahl Index to measure concentration. Antitrust Bull. 1982;27:285.

9. Calkins S. The new merger guidelines and the HerfindahlHirschman index. Cal L Rev. 1983;71:402.

10. Dave CV, Kesselheim AS, Fox ER, Qiu P, Hartzema A. High generic drug prices and market competition: a retrospective cohort study. Ann Intern Med. 2017;167:145-51.

11. Cutler DM, Scott Morton F. Hospitals, market share, and consolidation. JAMA. 2013;310(18):1964-70.

12. Schelman WR, Paulson AP, Aguilar K, Clark J, Cui ZL, Liepa AM, Hess LM. Predictive factors associated with ramucirumab monotherapy or combination therapy among patients with gastric/ gastroesophageal junction cancer in the community oncology setting. Ann Oncol 2017;28(Suppl 3):77.

13. Nordstrom BL, Simeone JC, Malley KG, Fraeman KH, Klippel Z, Durst M, Page JH, Xu H. Validation of claims algorithms for progression to metastatic cancer in patients with breast, non-small cell lung, and colorectal cancer. Front Oncol. 2016;6:18.

14. Nekhlyudov L, Levit L, Hurria A, Ganz PA. Patient-centered, evidence-based, and cost-conscious cancer care across the continuum: translating the Institute of Medicine report into clinical practice. CA Cancer J Clin. 2014;64(6):408-21. 